\title{
Correction: MYC amplifications in myeloma cell lines: correlation with MYC-inhibitor efficacy
}

\author{
Toril Holien $^{1}$, Kristine Misund ${ }^{1}$, Oddrun Elise Olsen ${ }^{1}$, Katarzyna Anna Baranowska ${ }^{1}$, \\ Glenn Buene ${ }^{1}$, Magne Børset ${ }^{1,2}$, Anders Waage ${ }^{1,3}$ and Anders Sundan ${ }^{1,4}$ \\ ${ }^{1}$ K.G. Jebsen Center for Myeloma Research, Department of Cancer Research and Molecular Medicine, Norwegian University \\ of Science and Technology, Trondheim, Norway \\ ${ }^{2}$ Department of Immunology and Transfusion Medicine, St. Olav's University Hospital, Trondheim, Norway \\ ${ }^{3}$ Department of Hematology, St. Olav's University Hospital, Trondheim, Norway \\ ${ }^{4}$ CEMIR (Centre of Molecular Inflammation Research), Department of Cancer Research and Molecular Medicine, Norwegian \\ University of Science and Technology, Trondheim, Norway \\ Published: November 13, 2018
}

Copyright: Holien et al. This is an open-access article distributed under the terms of the Creative Commons Attribution License 3.0 (CC BY 3.0), which permits unrestricted use, distribution, and reproduction in any medium, provided the original author and source are credited.

This article has been corrected: The legends for figures 1 and 2 were accidentally switched. The corrected figure legends are given below:

Figure 1: Expression of MYC in myeloma cell lines correlated positively with sensitivity to MYC inhibition. The IC50values of the MYC inhibitor 10058-F4 calculated from the results shown in Supplementary Figure 2 was compared with A. MYC mRNA values or B. MYC/GAPDH relative protein levels. The slope and $\mathrm{R}^{2}$-values are shown in the plots.

Figure 2: MYC gene copy numbers determine expression of MYC mRNA and protein in myeloma cell lines. In a panel of myeloma cell lines the levels of $M Y C$ gene copy numbers as measured by PCR was related to A. MYC mRNA measured using nCounter, and B. MYC protein levels measured using immunoblotting and normalized to GAPDH. The slope and $\mathrm{R}^{2}$-values are shown in the plots.

Original article: Oncotarget. 2015; 6:22698-22705. https://doi.org/10.18632/oncotarget.4245 